\title{
Circular RNA hsa_circ_0005114-miR-142-3p/miR-590-5p- adenomatous polyposis coli protein axis as a potential target for treatment of glioma
}

\author{
$\mathrm{BO} \mathrm{WEI}^{1 *}, \mathrm{LE} \mathrm{WANG}^{2 *}$ and JINGWEI ZHAO ${ }^{1}$ \\ ${ }^{1}$ Department of Neurosurgery, China-Japan Union Hospital of Jilin University, Changchun, Jilin 130033; \\ ${ }^{2}$ Department of Ophthalmology, The First Hospital of Jilin University, Jilin University, Changchun, Jilin 130021, P.R. China
}

Received September 12, 2019; Accepted October 22, 2020

DOI: $10.3892 / \mathrm{ol} .2020 .12320$

\begin{abstract}
Glioma is the most common type of brain tumor and is associated with a high mortality rate. Despite recent advances in treatment options, the overall prognosis in patients with glioma remains poor. Studies have suggested that circular (circ)RNAs serve important roles in the development and progression of glioma and may have potential as therapeutic targets. However, the expression profiles of circRNAs and their functions in glioma have rarely been studied. The present study aimed to screen differentially expressed circRNAs (DECs) between glioma and normal brain tissues using sequencing data collected from the Gene Expression Omnibus database (GSE86202 and GSE92322 datasets) and explain their mechanisms based on the competing endogenous (ce)RNA regulatory hypothesis. In total, 424 commonly downregulated DECs (with the Gene_symbol annotated in the circBase database) in these two datasets were identified. Using the CircInteractome and Starbase databases, 18 micro (mi)RNAs (miRs) were predicted to interact with DECs, while 22 glioma-related genes obtained from the Comparative Toxicogenomics Database were predicted to be regulated by 15 miRNAs via the miRwalk 2.0 database. A ceRNA network was established based on 115 DECs, 15 miRNAs and 22 mRNAs. LinkedOmics online analysis using The Cancer Genome Atlas (TCGA) data showed that hsa-miR-142-3p/hsa-miR-590-5p and their target gene adenomatous polyposis coli protein (APC) were all significantly associated with overall survival rate and their prognosis trend was opposite, revealing that high expression levels of hsa-miR-142-3p/hsa-miR-590-5 were associated with a poor overall survival rate, while high
\end{abstract}

Correspondence to: Dr Jingwei Zhao, Department of Neurosurgery, China-Japan Union Hospital of Jilin University, 126 Xiantai Street, Changchun, Jilin 130033, P.R. China

E-mail: jingweizhao726@aliyun.com

${ }^{*}$ Contributed equally

Key words: glioma, circular RNAs, competitive endogenous RNA, prognosis
APC expression with a good overall survival rate. UALCAN analysis using TCGA data of glioblastoma multiforme and the GSE25632 and GSE103229 microarray datasets showed that hsa-miR-142-3p/hsa-miR-590-5p was upregulated and APC was downregulated. Thus, hsa-miR-142-3p/hsa-miR-590-5pAPC-related circ/ceRNA axes may be important in glioma, and hsa_circ_0005114 interacted with both of these miRNAs. Functional analysis showed that hsa_circ_0005114 was involved in insulin secretion, while APC was associated with the Wnt signaling pathway. In conclusion, hsa_circ_0005114miR-142-3p/miR-590-5p-APC ceRNA axes may be potential targets for the treatment of glioma.

\section{Introduction}

Glioma is the most common malignant primary brain tumor, with an incidence of 4.7-7.3/100,000 individuals (1-4) and a mortality rate of 1.2-4.0/100,000 individuals (5). Despite advances in treatment options (such as radical surgery and chemo-radiotherapy), the prognosis of most patients remains poor due to high recurrence rates and invasiveness, with the 5-year survival rate of only 3-6\% for grade IV $(1,4)$. Therefore, it is important to further resolve the mechanisms underlying glioma progression to identify novel targets and develop new therapeutic approaches.

Circular (circ)RNAs are a newly identified class of non-coding (nc)RNAs with a covalent loop structure lacking the 5'-end cap and 3'-end ploy A tail, which prevents degradation by RNA exonucleases and confers strong stability in specific cells, such as tumor cells (6-8). Therefore, abnormal expression of circRNAs may be a potential mechanism for the development and progression of cancer, including glioma. This hypothesis has been demonstrated in several studies. For example, Zhou et al (9) demonstrated that hsa_circ_0008344 was significantly upregulated in glioblastoma tissues compared with the adjacent normal brain tissue. Zhou et al also reported that knockdown of hsa_circ_0008344 suppressed glioblastoma cell proliferation, colony formation, migration and invasion, but facilitated apoptosis. Wang et al (10) found that hsa circ_0001649 expression was decreased in glioma specimens and cell lines. In addition, downregulated hsa_circ_0001649 is significantly associated with advanced grade and poor prognosis for patients with glioma. The functions and mechanism of 
circRNAs remain unclear; however, accumulating evidence indicates circRNAs have micro (mi)RNA (miR) response elements (MREs) (6-8), while MREs are widely known to be located in the 3'-untranslated region (3'-UTR) of the target mRNAs and miRNAs inhibit mRNA translation by binding to MREs. Thus, circRNAs may directly bind to miRNAs, impacting the interaction between miRNAs and mRNAs and contributing to the progression of glioma. This is known as the competitive endogenous RNA (ceRNA) hypothesis (6-8). This mechanism of circRNA function has been reported in several studies. For example, circ-pituitary homeobox (PITX)1 is upregulated in cancerous tissues and four cell lines of glioma. Dual-luciferase reporter and rescue assays indicated that circ-PITX1 may exert its oncogenic functions by sponging miR-518a-5p, leading to the release of the miR-518a-5p-mediated repression of interleukin 17 receptor D (IL17RD) (11). Similarly, hsa_circ_0034642 levels are also increased in glioma tissues and cells. Mechanistically, hsa_circ_0034642 sponges miR-1205 to regulate basic leucine zipper ATF-like transcription factor (BATF)3 levels to facilitate cell proliferation, migration and invasion (12). Circ-epididymis-specific $\alpha$-mannosidase (MAN2B2) regulates S100 calcium-binding protein A8 (S100A8) expression by inhibiting miR-1205 and increasing S100A8 expression rescues the tumor suppressor effects of knockout of circ-MAN2B2 (13). Upregulated hsa_circ_0074362 plays a role in glioma progression by regulating the miR-1236-3p/homeobox B7 pathway (14), and downregulated hsa_circ_0001946 may act as a ceRNA, inhibiting glioblastoma progression by modulating miR-671-5p and cerebellar degeneration-related protein 1 (15). Overall, these findings imply that circRNA/ceRNA axes may be potential targets for the treatment of glioma; however, few studies have investigated this.

The present study aimed to further screen crucial circRNA/ceRNA axes for glioma by using the circRNA sequencing data of Yuan et al (16) and Zhu et al (17). Only the common differentially expressed circRNAs (DECs) in these two datasets were used to construct the ceRNA network based on the interactions between circRNAs-miRNAs and miRNAs-mRNAs. The expression and prognostic ability of these hub miRNAs and mRNAs were also validated using The Cancer Genome Atlas (TCGA) database and microarray data, which indirectly illustrated the possible proto- or antioncogenic roles of circRNAs. The current study may provide some novel therapeutic targets for glioma.

\section{Materials and methods}

Data collection. In total, two circRNA datasets of glioblastoma with the accession numbers GSE86202 (16) and GSE92322 (17) were downloaded from the Gene Expression Omnibus (GEO) database (http://www.ncbi.nlm.nih.gov/geo/). Three glioma and paired normal brain tissues were used for RNA sequencing on Illumina HiSeq 2500 (Homo sapiens, GPL16791) in GSE86202 dataset (16), while 10 samples (five glioma and paired normal brain tissues) were included for circRNA sequencing on Illumina HiSeq 2000 (Homo sapiens, GPL11154) in GSE92322 dataset (17).

Screening of DECs. The normalized data of each dataset was downloaded from the GEO database. The gene symbol and
circID of circRNAs were annotated using the circBase database (http://www.circbase.org) (18) according to the chromosome location and information of the positive-negative chain. The DECs were identified using edgeR package (http://www.bioconductor.org/packages/release/bioc/html/edgeR.html) (19). $\mid \log _{2} \mathrm{FC}$ (fold-change) $\mid>1$ and $\mathrm{P}<0.05$ were defined as the significance threshold values. The heatmap of DECs was created using pheatmap package (version 1.0.8; https://cran.r-project. $\mathrm{org} /$ web/packages/pheatmap) of R language (version 3.4.1; http://www.r-project.org/) (20).

Construction of circRNA-miRNA-mRNA ceRNA network. The miRNAs that interacted with common DECs in two datasets were predicted using the CircInteractome (https://circinteractome.nia.nih.gov) (21) and StarBase (http://starbase.sysu. edu.cn/starbase2/) (22) databases. Only the shared miRNAs predicted in these two databases were used for further analysis. The target genes of these miRNAs were subsequently predicted using the miRwalk 2.0 database (23), which included 12 prediction programs (miRWalk, MicroT4, miRanda, miRBridge, miRDB, miRMap, miRNAMap, PICTAR2, PITA, RNA22, RNAhybrid and Targetscan). Only the target genes predicted by at least six algorithms were retained, which were then compared with established glioma-related genes collected from the Comparative Toxicogenomics database (CTD) (24) to obtain glioma-associated miRNA-mRNA interaction pairs. The circRNA-miRNA-mRNA-ceRNA network was then established based on these interaction relationships between DECs-miRNAs and miRNAs-mRNAs using Cytoscape software (version 3.6.1; www.cytoscape.org/) (25).

Functional enrichment analysis for DECs and target genes in the ceRNA network. Enrichment analyses for Kyoto Encyclopedia of Genes and Genomes (KEGG) pathways and Gene Ontology (GO) [including biological process (BP), cellular component (CC) and molecular function (MF)] terms were conducted using the ClusterProfiler tool (26) to predict the possible function of DECs and target genes in the ceRNA network. $\mathrm{P}<0.05$ adjusted for multiple testing according to Benjamini and Hochberg (27) was regarded as the cut-off value.

Prognosis and expression validation for miRNAs and target genes in the ceRNA network. The LinkedOmics online database (http://www.linkedomics.org/) (28) was searched to explore the associations between miRNAs/mRNAs and overall survival (OS) rate for glioma based on a Cox regression, after which Kaplan-Meier survival curves were automatically provided. TCGA-glioblastoma multiforme-low grade glioma (GBMLGG) miRNASeq/RNA-seq and clinical data were selected for miRNAs/mRNAs analysis. The UALCAN web-portal (http://ualcan.path.uab.edu/index. html) (29) was used for validation of the expression of target genes of miRNAs between unmatched glioblastoma multiforme and normal control tissues using TCGA data, in which the statistical significance was estimated using unpaired Student's t-tests. GSE103229 (including five glioblastoma and five normal control tissues) and GSE25632 (including 82 glioblastoma and five normal controls) (30) microarray datasets in the GEO database were used to determine the expression 
Table I. circRNAs identified for glioma.

\begin{tabular}{llllll}
\hline & & \multicolumn{2}{c}{ GSE92322 } & & \multicolumn{2}{c}{ GSE86202 } \\
\cline { 3 - 5 } circID & Gene_symbol & logFC & P-value & logFC & P-value \\
\hline hsa_circ_0001368 & KLHL24 & -7.03946 & $1.62 \times 10^{-7}$ & -11.743 & $3.62 \times 10^{-3}$ \\
hsa_circ_0098551 & YAF2 & -6.11209 & $1.81 \times 10^{-4}$ & -9.32971 & $1.72 \times 10^{-2}$ \\
hsa_circ_0100496 & DGKH & -5.23572 & $6.47 \times 10^{-3}$ & -8.82079 & $2.37 \times 10^{-2}$ \\
hsa_circ_0001367 & KLHL24 & -5.88609 & $5.50 \times 10^{-4}$ & -10.9425 & $6.07 \times 10^{-3}$ \\
hsa_circ_0064615 & SLC4A7 & -5.87691 & $3.79 \times 10^{-4}$ & -10.562 & $7.75 \times 10^{-3}$ \\
hsa_circ_0060425 & PTPRT & -5.01633 & $1.54 \times 10^{-2}$ & -7.7421 & $4.77 \times 10^{-2}$ \\
hsa_circ_0001936 & BRWD3 & -5.02396 & $1.54 \times 10^{-2}$ & -8.73538 & $2.51 \times 10^{-2}$ \\
hsa_circ_0104727 & SH3GL3 & -5.67125 & $7.75 \times 10^{-4}$ & -11.395 & $4.53 \times 10^{-3}$ \\
hsa_circ_0005114 & RIMS2 & -8.09913 & $3.48 \times 10^{-11}$ & -6.44629 & $3.90 \times 10^{-2}$ \\
hsa_circ_0104726 & SH3GL3 & -4.80592 & $1.18 \times 10^{-2}$ & -10.2547 & $9.46 \times 10^{-3}$ \\
hsa_circ_0000120 & MAN1A2 & -4.33712 & $3.20 \times 10^{-2}$ & -11.2985 & $4.83 \times 10^{-3}$ \\
hsa_circ_0069718 & DCUN1D4 & -5.42038 & $2.74 \times 10^{-3}$ & -10.1942 & $9.83 \times 10^{-3}$ \\
hsa_circ_0130887 & PEX3 & -5.26036 & $6.47 \times 10^{-3}$ & -10.2911 & $9.24 \times 10^{-3}$ \\
hsa_circ_0004516 & C12orf24 & -4.78292 & $1.18 \times 10^{-2}$ & -9.66325 & $1.38 \times 10^{-2}$ \\
hsa_circ_0117841 & SLC4A10 & -5.46976 & $2.74 \times 10^{-3}$ & -10.8846 & $6.30 \times 10^{-3}$ \\
hsa_circ_0001369 & KLHL24 & -5.94584 & $2.61 \times 10^{-4}$ & -10.6354 & $7.40 \times 10^{-3}$ \\
\hline
\end{tabular}

Only the top 16 most significantly downregulated circRNAs that may act as a sponge of miR-142-3p/miR-590-5p are listed. FC, fold change; circ, circular.
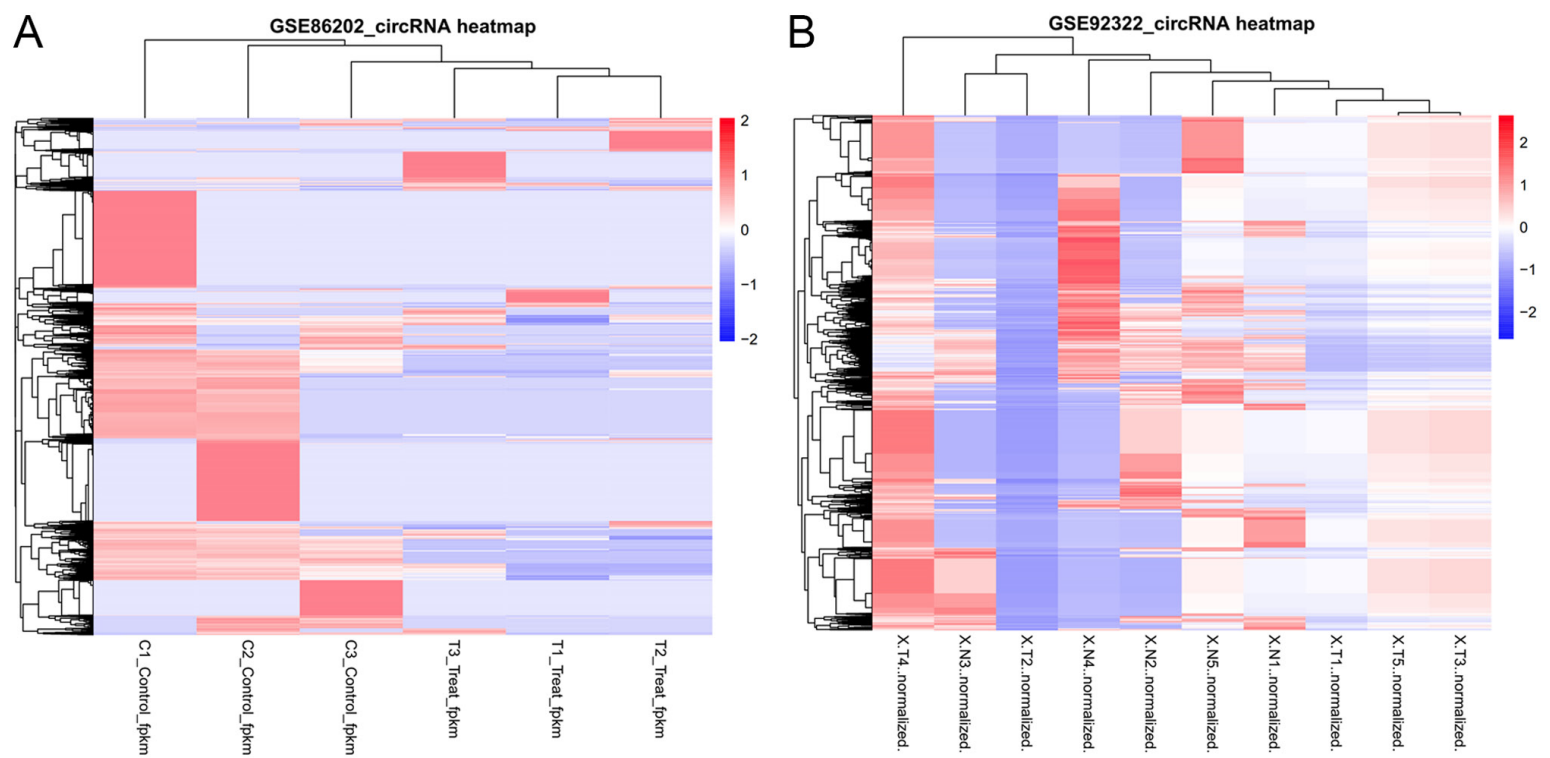

Figure 1. Heat map analysis of differentially expressed circRNAs in the (A) GSE86202 and (B) GSE92322 datasets. circ, circular.

of crucial miRNAs with GEO2R, an interactive web-based statistical tool (https://www.ncbi.nlm.nih.gov/geo/geo2r/). $\mathrm{P}<0.05$ was considered to indicate a statistically significant difference.

\section{Results}

Identification of DECs. In the GSE86202 dataset, 23,463/37,085 circRNAs were annotated to the circID in the circBase database and 6,131 had corresponding Gene_symbols. In the
GSE92322 dataset, 12,863/59,386 circRNAs were annotated to the circID in the circBase database and 4,676 of them had corresponding Gene_symbol.

Using the edgeR package, a total of 22 upregulated (20 had circID and Gene_symbol) and 2,068 (1,874 had circID and 1,332 had Gene_symbol) DECs were identified between glioma and paired normal brain tissues in the GSE86202 dataset. Meanwhile, 29 upregulated (zero had circID and Gene_symbol) and 1,205 (1,196 had circID and 884 had Gene_symbol) DECs were identified in the GSE92322 dataset (Table I). The heat maps 
Table II. Kyoto Encyclopedia of Genes and Genomes pathways enriched for differentially expressed circRNAs.

\begin{tabular}{|c|c|c|c|}
\hline ID & Description & $\begin{array}{l}\text { Adjusted } \\
\text { P-value }\end{array}$ & Gene ID \\
\hline hsa04723 & $\begin{array}{l}\text { Retrograde endocannabinoid } \\
\text { signaling }\end{array}$ & $1.65 \times 10^{-2}$ & $\begin{array}{l}\text { CACNA1A/CACNA1C/GABRB2/GABRG3/GNAQ/ } \\
\text { NAPEPLD/NDUFA10/NDUFS1/PRKACB/PRKCB/RIMS1 }\end{array}$ \\
\hline hsa04012 & ErbB signaling pathway & $1.65 \times 10^{-2}$ & AKT2/AKT3/PAK1/PAK3/PIK3CA/PRKCB/PTK2/SOS2 \\
\hline hsa04911 & Insulin secretion & $1.65 \times 10^{-2}$ & $\begin{array}{l}\text { CACNA1C/GNAQ/KCNMA1/PCLO/PRKACB/PRKCB/ } \\
\text { RIMS2/RYR2 }\end{array}$ \\
\hline hsa04020 & Calcium signaling pathway & $1.65 \times 10^{-2}$ & $\begin{array}{l}\text { RYR2/CACNA1A/CACNA1C/CACNA1E/CAMK4/GNAQ/ } \\
\text { PDE1C/PHKB/PRKACB/PRKCB/RYR2/SLC8A1 }\end{array}$ \\
\hline hsa04725 & Cholinergic synapse & $1.65 \times 10^{-2}$ & $\begin{array}{l}\text { AKT2/AKT3/CACNA1A/CACNA1C/CAMK4/GNAQ/ } \\
\text { PIK3CA/PRKACB/PRKCB }\end{array}$ \\
\hline hsa05211 & Renal cell carcinoma & $1.65 \times 10^{-2}$ & AKT2/AKT3/CREBBP/PAK1/PAK3/PIK3CA/SOS2 \\
\hline hsa05205 & Proteoglycans in cancer & $2.22 \times 10^{-2}$ & $\begin{array}{l}\text { AKT2/AKT3/ANK2/ANK3/ARHGEF12/PAK1/PIK3CA/ } \\
\text { PRKACB/PRKCB/PTK2/SOS2/TIAM1 }\end{array}$ \\
\hline hsa05231 & Choline metabolism in cancer & $2.27 \times 10^{-2}$ & AKT2/AKT3/DGKB/DGKH/DGKI/PIK3CA/PRKCB/SOS2 \\
\hline hsa04660 & $\mathrm{T}$ cell receptor signaling pathway & $2.30 \times 10^{-2}$ & AKT2/AKT3/DGKI/4CACNA1C/PAK1/PAK3/PIK3CA/SOS2 \\
\hline hsa04810 & Regulation of actin cytoskeleton & $2.58 \times 10^{-2}$ & $\begin{array}{l}\text { APC/ARHGEF12/ARHGEF7/DOCK1/MYH10/PAK1/PAK3/ } \\
\text { PIK3CA/PIKFYVE/PTK2/SOS2/TIAM1 }\end{array}$ \\
\hline hsa04024 & cAMP signaling pathway & $3.38 \times 10^{-2}$ & $\begin{array}{l}\text { AKT2/AKT3/RYR2/CACNA1C/CAMK4/CREBBP/PAK1/ } \\
\text { PIK3CA/PRKACB/RYR2/TIAM1 }\end{array}$ \\
\hline hsa04720 & Long-term potentiation & $3.38 \times 10^{-2}$ & CACNA1C/CAMK4/CREBBP/GNAQ/PRKACB/PRKCB \\
\hline hsa04919 & $\begin{array}{l}\text { Thyroid hormone signaling } \\
\text { pathway }\end{array}$ & $3.38 \times 10^{-2}$ & $\begin{array}{l}\text { AKT2/AKT3/CREBBP/MED13L/PIK3CA/PRKACB/ } \\
\text { PRKCB/THRB }\end{array}$ \\
\hline hsa05032 & Morphine addiction & $3.38 \times 10^{-2}$ & $\begin{array}{l}\text { CACNA1A/GABRB2/GABRG3/PDE1C/PDE8A/PRKACB/ } \\
\text { PRKCB }\end{array}$ \\
\hline hsa04014 & Ras signaling pathway & $3.38 \times 10^{-2}$ & $\begin{array}{l}\text { AKT2/AKT3/NTRK2/PAK1/PAK3/PIK3CA/PRKACB/ } \\
\text { PRKCB/RAPGEF5/RASA2/SOS2/TIAM1 }\end{array}$ \\
\hline hsa04961 & $\begin{array}{l}\text { Endocrine and other factor-regulated } \\
\text { calcium reabsorption }\end{array}$ & $3.38 \times 10^{-2}$ & RYR2/GNAQ/PRKACB/PRKCB/SLC8A1 \\
\hline hsa00280 & $\begin{array}{l}\text { Valine, leucine and isoleucine } \\
\text { degradation }\end{array}$ & $3.48 \times 10^{-2}$ & HADHB/HMGCLL1/HMGCS1/MCCC1/PCCA \\
\hline hsa00510 & $\mathrm{N}$-Glycan biosynthesis & $3.48 \times 10^{-2}$ & FUT8/MAN1A2/MAN2A1/ST6GAL2/TUSC3 \\
\hline hsa04662 & B cell receptor signaling pathway & $3.48 \times 10^{-2}$ & AKT2/AKT3/4CACNA1C/PIK3CA/PRKCB/SOS2 \\
\hline hsa05170 & $\begin{array}{l}\text { Human immunodeficiency virus } \\
1 \text { infection }\end{array}$ & $3.94 \times 10^{-2}$ & $\begin{array}{l}\text { AKT2/AKT3/CUL5/GNAQ/4CACNA1C/PAK1/PAK3/ } \\
\text { PDIA3/PIK3CA/PRKCB/PTK2 }\end{array}$ \\
\hline hsa04070 & $\begin{array}{l}\text { Phosphatidylinositol signaling } \\
\text { system }\end{array}$ & $3.94 \times 10^{-2}$ & DGKB/DGKH/DGKI/PIK3C3/PIK3CA/PIKFYVE/PRKCB \\
\hline hsa05214 & Glioma & $3.95 \times 10^{-2}$ & AKT2/AKT3/CAMK4/PIK3CA/PRKCB/SOS2 \\
\hline hsa04728 & Dopaminergic synapse & $4.67 \times 10^{-2}$ & $\begin{array}{l}\text { AKT2/AKT3/CACNA1A/CACNA1C/CLOCK/GNAQ/ } \\
\text { PRKACB/PRKCB }\end{array}$ \\
\hline
\end{tabular}

APC, adenomatous polyposis coli protein.

of these DECs are displayed in Fig. 1. These findings suggested that these DECs may be effective to classify the samples into two groups.

Functional enrichment analysis for DECs. A comparison between the two aforementioned datasets reported that 424 downregulated DECs with Gene_symbol were shared. The functions of DECs were enriched according to their corresponding Gene_symbols. The results showed that
23 KEGG pathways were enriched (Table II and Fig. 2A), mainly including 'retrograde endocannabinoid signaling', 'ErbB signaling pathway' and 'insulin secretion' for hsa_circ_0005114. Meanwhile, KEGG pathways enriched for hsa_circ_0100496 were 'calcium signaling pathway', 'cholinergic synapse' and 'choline metabolism in cancer', and 'N-Glycan biosynthesis' was enriched for hsa_circ_0000120. Furthermore, $143 \mathrm{GO}$ terms (including $87 \mathrm{BPs}, 38 \mathrm{CCs}$ and 18 MFs; Table III and Fig. 2B) were also enriched, including 
A

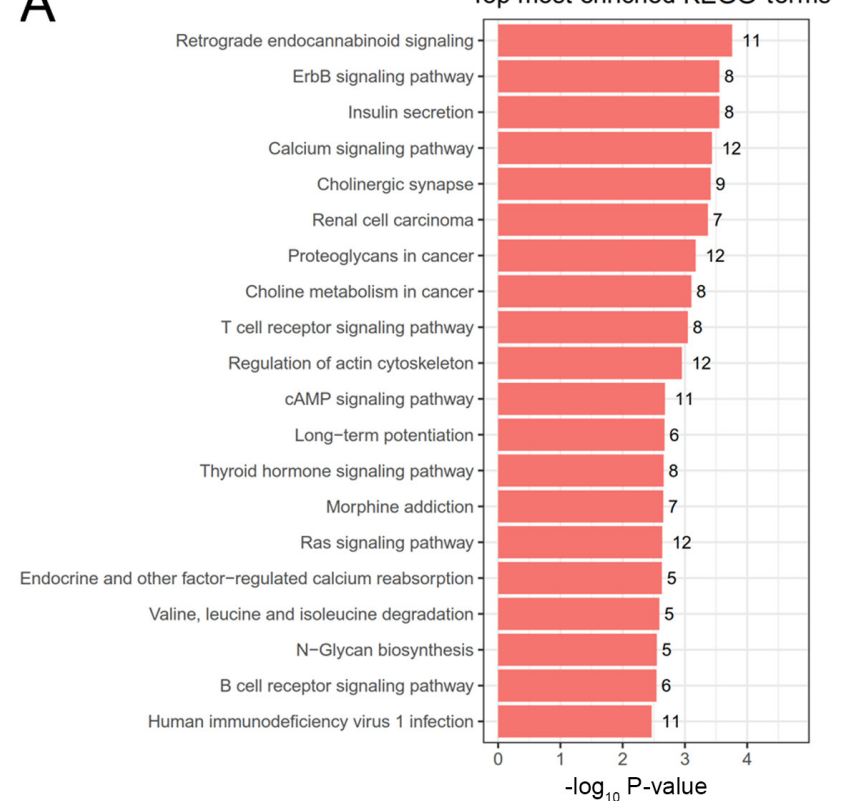

B

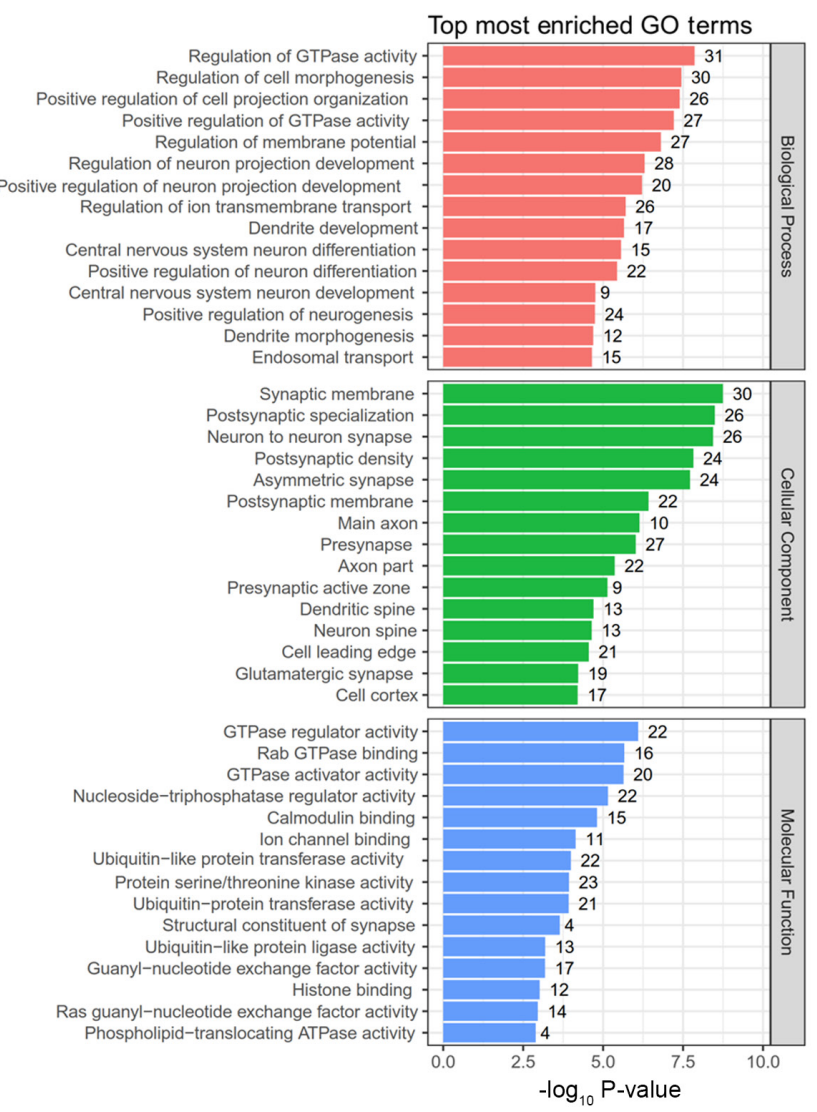

Figure 2. Function enrichment analysis for differentially expressed circular RNAs. (A) Top 20 KEGG pathways and (B) top 15 biological process, cellular component and molecular function GO terms. KEGG, Kyoto Encyclopedia of Genes and Genomes; GO, Gene Ontology.

'regulation of cell morphogenesis' (hsa_circ_0005114 and hsa_circ_0001936), 'regulation of neuron projection development' (hsa_circ_0005114), 'central nervous system neuron differentiation' (hsa_circ_0117841), 'positive regulation of neuron differentiation' (hsa_circ_0005114, hsa_circ_0104727) and 'cell-cell junction' (hsa_circ_0001368). These findings suggested that hsa_circ_0005114 may be crucial for the development of glioma since it was enriched in KEGG pathways and GO terms.

Construction of the ceRNA network and functional enrichment. After uploading the circID (197 common in the two datasets) to the CircInteractome database, 313 miRNAs were predicted to interact with DECs. Using Gene_symbol, 276 miRNAs were predicted to interact with DECs using Starbase. Among them, 18 miRNAs were shared, including hsa-miR-139-5p, -142-3p, -184, -217, -324-5p, -326, -330-5p, $-338-3 p,-346,-375,-384,-421,-496,-543,-590-5 p,-599$, $-615-3 p$ and $-875-5 p)$. Subsequently, the target genes were predicted for these 18 miRNAs using the miRwalk 2.0 database, which were then compared with 65 pre-established glioma-related genes collected from the CTD database. In total, 22 genes were shared, including BRD4, SUZ12, VEGFA, NOTCH3, NOTCH2, RUNX3, CTNND2, EGFR, CDK6, PML, BHLHE40, EGF, NOTCH1, TNFSF10, LZTR1, RUNX1, MDM4, MET, IL1B, JAG1, APC and RECK. Then, the ceRNA network was established based on 115 DECs, 15 miRNAs and the aforementioned 22 mRNAs (Fig. 3).

Function analysis for the target genes of miRNAs in this ceRNA network showed that 44 KEGG pathways (Table IV and Fig. 4A) and 736 GO terms (including 713 BPs, three CCs and $20 \mathrm{MFs}$ ) (Table V and Fig. 4B) were enriched, such as 'regulation of actin cytoskeleton' (Table IV), various cancer pathways (including 'miRNAs in cancer', 'breast cancer', 'endometrial cancer', 'gastric cancer', 'hepatocellular carcinoma' and 'colorectal cancer'; Fig. 4A), 'Wnt signaling pathway', 'cell cycle arrest', 'cell fate commitment' (Fig. 4B) and cell junction assembly (Table V), in all of which the APC gene was included. These findings suggested that the circRNAs-miRNAs that regulated APC may be crucial for the development of glioma.

Prognosis and expression analysis for miRNAs and target genes. LinkedOmics online analysis using TCGA data showed that 19 mRNAs and nine miRNAs were significantly associated with OS (Table VI and Fig. 5). These prognosis-related miRNAs and mRNAs constituted 20 interaction relationships in the ceRNA network. However, the prognosis trend between hsa-miR-217/hsa-miR-324-5p/hsa-miR-590-5p/hsa-miR-599/ hsa-miR-875-5p and their target genes were consistent, with high expression levels of both miRNAs and their target genes indicating a poor prognosis, which is not in accordance with the negative regulatory mechanisms of miRNAs on mRNAs. Thus, only hsa-miR-139-5p-NOTCH1/RUNX1/CDK6/ TNFSF10/VEGFA and hsa-miR-142-3p-APC-related ceRNA axes may be important. Furthermore, as all the circRNAs in the ceRNA network were downregulated, its interactive miRNAs should be upregulated according to the ceRNA theory (6-8); however, similarly to circRNAs, high expression levels of hsa-miR-139-5p were also associated with an excellent overall survival rate, indicating that hsa-miR-139-5p was also downregulated in glioma. Thus, hsa-miR-139-5p-related interaction relationships were excluded from further analyses and only hsa-miR-142-3p-APC-related ceRNA axes (in which high hsa-miR-142-3p expression was associated with poor OS, while high APC expression was associated with a good prognosis, as shown in Fig. 5) were crucial. 
Table III. Significant GO terms enriched for differentially expressed circRNAs.

\begin{tabular}{|c|c|c|c|c|}
\hline $\begin{array}{l}\mathrm{GO} \\
\text { term }\end{array}$ & ID & Description & $\begin{array}{l}\text { Adjusted } \\
\text { P-value }\end{array}$ & Gene ID \\
\hline $\mathrm{BP}$ & GO:0022604 & $\begin{array}{l}\text { Regulation of cell } \\
\text { morphogenesis }\end{array}$ & $4.70 \times 10^{-5}$ & $\begin{array}{l}\text { ARHGAP44/ARHGEF7/BRWD1/BRWD3/CAPRIN1/ } \\
\text { CORO1C/CUX1/DLG1/DOCK1/FMNL2/HECW1/ } \\
\text { HECW2/MKLN1/MYH10/NTRK2/PAK1/PAK3/ } \\
\text { PARP6/PHIP/PSEN1/PTK2/RHOBTB3/RIMS1/RIMS2/ } \\
\text { ROBO1/ROBO2/SYT1/TIAM1/TNIK/ZMYM4 }\end{array}$ \\
\hline $\mathrm{BP}$ & GO:0031346 & $\begin{array}{l}\text { Positive regulation of cell } \\
\text { projection organization }\end{array}$ & $4.70 \times 10^{-5}$ & $\begin{array}{l}\text { APC/ARHGEF7/ATP8A2/AUTS2/CAPRIN1/CBFA2T2/ } \\
\text { CCDC88A/CORO1C/CPEB3/CUX1/HTT/KDM1A/ } \\
\text { LRRC7/MAGI/NTRK2/PAK1/PAK3/PARP6/PSEN1/ } \\
\text { RIMS1/RIMS2/ROBO1/ROBO2/SYT1/TENM3/ } \\
\text { TIAM1 }\end{array}$ \\
\hline BP & GO:0042391 & $\begin{array}{l}\text { Regulation of membrane } \\
\text { potential }\end{array}$ & $1.09 \times 10^{-4}$ & $\begin{array}{l}\text { AKAP6/AKT2/ANK2/ANK3/APP/CACNA1A/ } \\
\text { CACNA1C/CACNA1E/DGKI/DLG1/FGF14/GABRB2/ } \\
\text { GABRG3/GCLM/GNAQ/KCNH1/KCNK2/KCNMA1/ } \\
\text { NALCN/NDUFS1/NTRK2/PSEN1/RIMS1/RIMS2/ } \\
\text { RYR2/SLC8A1/SLMAP }\end{array}$ \\
\hline BP & GO:0010975 & $\begin{array}{l}\text { Regulation of neuron } \\
\text { projection development }\end{array}$ & $2.93 \times 10^{-4}$ & $\begin{array}{l}\text { ARHGAP44/ATP8A2/CAMSAP2/CAPRIN1/CBFA2T2/ } \\
\text { CCDC88A/CPEB3/CUX1/FBXO7/HECW1/HECW2/ } \\
\text { KDM1A/LRRC7/MAGI2/NTRK2/PAK1/PAK3/PARP6/ } \\
\text { PSEN1/PTK2/RIMS1/RIMS2/ROBO1/ROBO2/SYT1/ } \\
\text { TENM3/TIAM1/TNIK }\end{array}$ \\
\hline $\mathrm{BP}$ & GO:0034765 & $\begin{array}{l}\text { Regulation of ion } \\
\text { transmembrane transport }\end{array}$ & $8.62 \times 10^{-4}$ & $\begin{array}{l}\text { AKAP6/AKT2/ANK2/ANK3/APP/ATG5/CACNA1A/ } \\
\text { CACNA1C/CACNA1E/CRBN/DLG1/FGF14/HECW1/ } \\
\text { HECW2/HTT/KCNH1/KCNMA1/KLHL24/NALCN/ } \\
\text { PKD2/PSEN1/RYR2/SLC8A1/SLMAP/STK39/ } \\
\text { UBQLN1 }\end{array}$ \\
\hline $\mathrm{BP}$ & GO:0021953 & $\begin{array}{l}\text { Central nervous system } \\
\text { neuron differentiation }\end{array}$ & $9.64 \times 10^{-4}$ & $\begin{array}{l}\text { ADARB1/AGTPBP1/DCLK1/DCLK2/GIGYF2/ } \\
\text { HERC1/LRP6/NFIB/NTRK2/PSEN1/PTK2/ROBO1/ } \\
\text { ROBO2/SATB2/SLC4A10 }\end{array}$ \\
\hline $\mathrm{BP}$ & GO:0045666 & $\begin{array}{l}\text { Positive regulation of neuron } \\
\text { differentiation }\end{array}$ & $1.17 \times 10^{-4}$ & $\begin{array}{l}\text { ATP8A2/CAPRIN1/CBFA2T2/CPEB3/CUX1/KDM1A/ } \\
\text { LRRC7/MAGI2/NTRK2/PAK1/PAK3/PARP6/PSEN1/ } \\
\text { RIMS1/RIMS2/ROBO1/ROBO2/SH3GL3/SYT1/TCF4/ } \\
\text { TENM3/TIAM1 }\end{array}$ \\
\hline BP & GO:0021954 & $\begin{array}{l}\text { Central nervous system } \\
\text { neuron development }\end{array}$ & $4.84 \times 10^{-3}$ & $\begin{array}{l}\text { ADARB1/DCLK1/DCLK2/NFIB/NTRK2/PTK2/ } \\
\text { ROBO1/ROBO2/SLC4A10 }\end{array}$ \\
\hline BP & GO:0050769 & $\begin{array}{l}\text { Positive regulation of } \\
\text { neurogenesis }\end{array}$ & $4.84 \times 10^{-3}$ & $\begin{array}{l}\text { APP/ATP8A2/CAPRIN1/CBFA2T2/CPEB3/CUX1/ } \\
\text { KDM1A/LRRC7/MAGI/MAN2A1/NTRK2/PAK1/ } \\
\text { PAK3/PARP6/PSEN1/RIMS1/RIMS2/ROBO1/ROBO2/ } \\
\text { SH3GL3/SYT1/TCF4/TENM3/TIAM1 }\end{array}$ \\
\hline $\mathrm{BP}$ & GO:0097479 & Synaptic vesicle localization & $5.75 \times 10^{-3}$ & $\begin{array}{l}\text { AP3B2/CADPS2/DGKI/ERC2/MAGI2/PCLO/PRKCB/ } \\
\text { PSEN1/RIMS1/RIMS2/STXBP5L/SYN3/SYT1 }\end{array}$ \\
\hline $\mathrm{CC}$ & GO:0097060 & Synaptic membrane & $5.96 \times 10^{-7}$ & $\begin{array}{l}\text { ANK2/ANK3/ANKS1B/ARHGAP32/ATP2B1/ } \\
\text { CACNA1C/CADPS2/CNKSR2/CPEB3/DENND1A/ } \\
\text { DGKI/DLG1/DLG2/ERC1/ERC2/GABRB2/GABRG3/ } \\
\text { KCNH1/KCNMA1/LRRC7/PICALM/PSD3/PSEN1/ } \\
\text { RIMS1/RIMS2/SLC8A1/STRN/SYNE1/SYT1/ } \\
\text { TIAM1 }\end{array}$ \\
\hline $\mathrm{CC}$ & GO:0099572 & Postsynaptic specialization & $5.96 \times 10^{-7}$ & $\begin{array}{l}\text { ANKS1B/ARHGAP32/ARHGAP44/CACNA1C/ } \\
\text { CNKSR2/CPEB3/DCLK1/DGKI/DLG1/DLG2/ } \\
\text { GABRB2/KCNH1/LRRC7/MAGI2/MIB1/NTRK2/ } \\
\text { PAK3/PCLO/PSD3/SEPT11/SH3GL3/STRN/SYN3/ } \\
\text { TANC2/TIAM1/TNIK }\end{array}$ \\
\hline
\end{tabular}


Table III. Continued.

\begin{tabular}{|c|c|c|c|c|}
\hline $\begin{array}{l}\text { GO } \\
\text { term }\end{array}$ & ID & Description & $\begin{array}{l}\text { Adjusted } \\
\text { P-value }\end{array}$ & Gene ID \\
\hline $\mathrm{CC}$ & GO:0098984 & Neuron to neuron synapse & $5.96 \times 10^{-7}$ & $\begin{array}{l}\text { ANKS1B/ARHGAP32/ARHGAP44/CACNA1C/ } \\
\text { CNKSR2/CPEB3/DCLK1/DGKI/DLG1/DLG2/KCNH1/ } \\
\text { LRRC7/MAGI/MIB1/NTRK2/PAK3/PCLO/PSD3/ } \\
\text { SEPT11/SH3GL3/STRN/SYN3/SYT1/TANC2/TIAM1/ } \\
\text { TNIK }\end{array}$ \\
\hline $\mathrm{CC}$ & GO:0014069 & Postsynaptic density & $1.84 \times 10^{-6}$ & $\begin{array}{l}\text { ANKS1B/ARHGAP32/ARHGAP44/CACNA1C/ } \\
\text { CNKSR2/CPEB3/DCLK1/DGKI/DLG1/DLG2/KCNH1/ } \\
\text { LRRC7/MAGI2/MIB1/NTRK2/PAK3/PCLO/PSD3/ } \\
\text { SH3GL3/STRN/SYN3/TANC2/TIAM1/TNIK }\end{array}$ \\
\hline $\mathrm{CC}$ & GO:0098793 & Presynapse & $5.86 \times 10^{-5}$ & $\begin{array}{l}\text { APP/ARHGAP44/ATP2B1/CADPS2/DENND1A/DGKI/ } \\
\text { ERC1/ERC2/HTT/ICA1/KCNH1/KCNK2/MCTP1/ } \\
\text { NTRK2/PCLO/PICALM/PRKCB/PSEN1/RIMS1/ } \\
\text { RIMS2/RPH3A/SH3GL3/STX12/SYN3/SYT1/TNIK/ } \\
\text { WDR7 }\end{array}$ \\
\hline $\mathrm{CC}$ & GO:0048786 & Presynaptic active zone & $3.58 \times 10^{-4}$ & $\begin{array}{l}\text { APP/ARHGAP44/ATP2B1/DGKI/ERC1/ERC2/PCLO/ } \\
\text { RIMS1/RIMS2 }\end{array}$ \\
\hline $\mathrm{CC}$ & GO:0098978 & Glutamatergic synapse & $2.05 \times 10^{-3}$ & $\begin{array}{l}\text { ARHGAP44/ATP2B1/CADPS2/CAMK4/CNKSR2/ } \\
\text { DGKB/DGKI/DLG1/DLG2/ERC2/MYH10/PAK3/ } \\
\text { SEPT11/SH3GL3/SYN3/SYT1/TANC2/TIAM1/TNIK }\end{array}$ \\
\hline $\mathrm{CC}$ & GO:0005938 & Cell cortex & $2.05 \times 10^{-3}$ & $\begin{array}{l}\text { AKT2/ARHGAP32/ARHGEF7/ERC2/EXOC6B/ } \\
\text { FMN2/MKLN1/MYH10/PCLO/PKD2/PSEN1/PTK2/ } \\
\text { RHOBTB3/RIC8B/RIMS1/RIMS2/SEPT11 }\end{array}$ \\
\hline $\mathrm{CC}$ & GO:0043025 & Neuronal cell body & $2.28 \times 10^{-3}$ & $\begin{array}{l}\text { AMFR/ARHGEF7/ATP2B1/CACNA1A/CACNA1C/ } \\
\text { CNKSR2/DENND1A/DGKI/GIGYF2/KCNH1/KCNK2/ } \\
\text { KLHL24/LMTK2/LRP6/MYH10/PDE1C/PICALM/ } \\
\text { PSEN1/SLC1A4/SLC4A10/STRN/TIAM1/TMEM50A }\end{array}$ \\
\hline $\mathrm{CC}$ & GO:0005911 & Cell-cell junction & $8.24 \times 10^{-3}$ & $\begin{array}{l}\text { AKAP6/ANK2/ANK3/APC/APP/ASH1L/DLG1/ } \\
\text { KLHL24/LRRC7/MAGI2/NFASC/PAK1/PIKFYVE/ } \\
\text { PKD2/SDCCAG8/SLC8A1/SPECC1L/STRN/TIAM1/ } \\
\text { TJP1 }\end{array}$ \\
\hline MF & GO:0017137 & Rab GTPase binding & $4.74 \times 10^{-4}$ & $\begin{array}{l}\text { ACAP2/CLEC16A/DENND1A/DENND1B/DENND5B/ } \\
\text { ERC1/GAPVD1/PICALM/RHOBTB3/RIMS1/RIMS2/ } \\
\text { RPH3A/STXBP5L/TBC1D12/TBC1D1/TBCK }\end{array}$ \\
\hline MF & GO:0044325 & Ion channel binding & $7.48 \times 10^{-3}$ & $\begin{array}{l}\text { AKAP6/ANK2/ANK3/DLG1/HTT/KCNH1/PKD2/ } \\
\text { RIMS1/RIMS2/RYR2/SLC8A1 }\end{array}$ \\
\hline MF & GO:0004143 & Diacylglycerol kinase activity & $4.94 \times 10^{-2}$ & DGKB/DGKH/DGKI \\
\hline
\end{tabular}

Only terms enriched by significantly differentially expressed circRNAs that may act as a sponge of miR-142-3p/miR-590-5p are listed. The genes enriched in each term were corresponding to differentially expressed circRNAs. GO, Gene Ontology; BP, biological process; CC, cellular component; MF, molecular function; circ, circular; APC, adenomatous polyposis coli protein.

Accordingly, hsa_circ_0000120/ 0001367/ 0001368/ 0001369/0001936/0004516/0005114/0060425/0064615/ 0130887-hsa-miR-142-3p-APC ceRNA axes may be potential targets for the development of glioma. Additionally, UALCAN analysis using TCGA data of glioblastoma multiforme demonstrated that APC was significantly downregulated in glioma compared with in normal tissues $(\mathrm{P}=0.0000822)$; while GSE25632 microarray analysis showed the expression of hsa-miR-142-3p was significantly higher in glioma compared with that in normal tissues $\left(\log _{2} \mathrm{FC}=1.21, \mathrm{P}=0.00284\right)$, further confirming the creditability of these crucial ceRNA axes for glioma (data not shown).

In addition, both of GSE25632 $\left(\log _{2} \mathrm{FC}=5.75, \mathrm{P}=0.0226\right)$ and GSE103229 $\left(\log _{2} \mathrm{FC}=1.65, \mathrm{P}=0.0208\right)$ microarray analyses revealed that hsa-miR-590-5p was upregulated in glioma compared with that in normal tissues (data not shown). In fact, the interaction between hsa-miR-590-5p and APC was also predicted by RNA22 and RNAhybrid using miRwalk2.0 analysis (data not shown). Therefore, circRNAs interacting with hsa-miR-590-5p were also important, including 


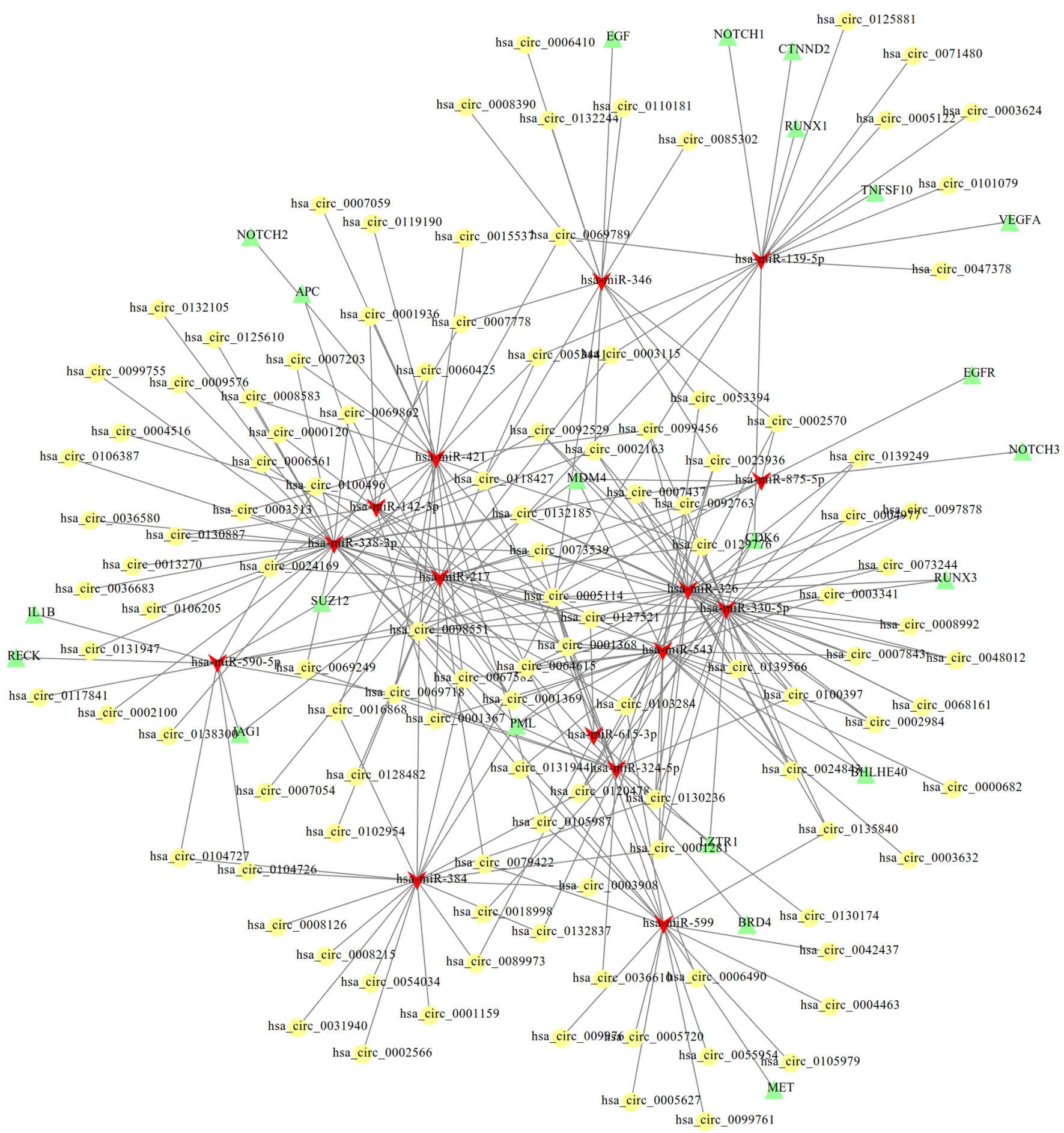

Figure 3. Competing endogenous RNA interaction network of circRNA-miRNA-mRNA. Yellow circles represent circRNAs, green triangles represent mRNAs and red inverted arrowheads represent miRNAs.

hsa_circ_0005114, 0069718, 0098551, 0100496, 0104726 , 0104727 and 0117841 (Fig. 3), among which hsa_circ_0005114 interacted with hsa-miR-142-3p. Therefore, the hsa circ_0005114-miR-142-3p/miR-590-5p-APC ceRNA axis may represent a potential mechanism for the development of glioma.

\section{Discussion}

In the present study, 16 crucial downregulated circRNAs were identified that may sponge $\mathrm{miR}-142-3 \mathrm{p} / \mathrm{miR}-590-5 \mathrm{p}$ to suppress glioma progression via regulating the APC gene. Most of them, to the best of our knowledge, were newly reported circRNAs associated with cancer, except for hsa_circ_0001368 that has a tumor-suppression role in gastric cancer by regulating the miR-6506-5p/forkhead box (FOX) protein O3 axis (31). However, hsa_circ_0005114 may be particularly important as it was a circRNA interacting with both miR-142-3p and miR-590-5p.

The present analysis did not identify the roles of hsa circ_0005114 in cancer; however, hsa_circ_0005114 is derived 
Table IV. Kyoto Encyclopedia of Genes and Genomes pathways enriched for genes in the competing endogenous RNA network.

\begin{tabular}{|c|c|c|c|}
\hline ID & Description & $\begin{array}{l}\text { Adjusted } \\
\text { P-value }\end{array}$ & Gene ID \\
\hline hsa05206 & microRNAs in cancer & $3.02 \times 10^{-8}$ & $\begin{array}{l}\text { APC/CDK6/EGFR/MDM4/MET/NOTCH1/ } \\
\text { NOTCH2/NOTCH3/RECK/VEGFA }\end{array}$ \\
\hline hsa05224 & Breast cancer & $3.50 \times 10^{-8}$ & $\begin{array}{l}\text { APC/CDK6/EGF/EGFR/JAG1/NOTCH1/NOTCH2/ } \\
\text { NOTCH3 }\end{array}$ \\
\hline hsa05165 & Human papillomavirus infection & $6.64 \times 10^{-7}$ & $\begin{array}{l}\text { APC/CDK6/EGF/EGFR/JAG1/NOTCH1/NOTCH2/ } \\
\text { NOTCH3/VEGFA }\end{array}$ \\
\hline hsa04658 & Th1 and Th2 cell differentiation & $4.91 \times 10^{-5}$ & JAG1/NOTCH1/NOTCH2/NOTCH3/RUNX3 \\
\hline hsa01522 & Endocrine resistance & $5.38 \times 10^{-5}$ & EGFR/JAG1/NOTCH1/NOTCH2/NOTCH3 \\
\hline hsa04330 & Notch signaling pathway & $7.36 \times 10^{-5}$ & JAG1/NOTCH1/NOTCH2/NOTCH3 \\
\hline hsa05218 & Melanoma & $3.22 \times 10^{-4}$ & CDK6/EGF/EGFR/MET \\
\hline hsa05212 & Pancreatic cancer & $3.31 \times 10^{-4}$ & CDK6/EGF/EGFR/VEGFA \\
\hline hsa01521 & EGFR tyrosine kinase inhibitor resistance & $3.62 \times 10^{-4}$ & EGF/EGFR/MET/VEGFA \\
\hline hsa05219 & Bladder cancer & $1.22 \times 10^{-3}$ & EGF/EGFR/VEGFA \\
\hline hsa05213 & Endometrial cancer & $3.13 \times 10^{-3}$ & APC/EGF/EGFR \\
\hline hsa05226 & Gastric cancer & $3.21 \times 10^{-3}$ & APC/EGF/EGFR/MET \\
\hline hsa05223 & Non-small cell lung cancer & $3.81 \times 10^{-3}$ & CDK6/EGF/EGFR \\
\hline hsa04010 & MAPK signaling pathway & $3.81 \times 10^{-3}$ & EGF/EGFR/RUNX3/MET/VEGFA \\
\hline hsa05225 & Hepatocellular carcinoma & $4.05 \times 10^{-3}$ & APC/CDK6/EGFR/MET \\
\hline hsa05214 & Glioma & $4.59 \times 10^{-3}$ & CDK6/EGF/EGFR \\
\hline hsa05210 & Colorectal cancer & $6.37 \times 10^{-3}$ & APC/EGF/EGFR \\
\hline hsa04510 & Focal adhesion & $6.37 \times 10^{-3}$ & EGF/EGFR/MET/VEGFA \\
\hline hsa04151 & PI3K-Akt signaling pathway & $6.44 \times 10^{-3}$ & CDK6/EGF/EGFR/MET/VEGFA \\
\hline hsa04015 & Rap1 signaling pathway & $6.52 \times 10^{-3}$ & EGF/EGFR/MET/VEGFA \\
\hline hsa04066 & HIF-1 signaling pathway & $8.06 \times 10^{-3}$ & EGF/EGFR/VEGFA \\
\hline hsa05163 & Human cytomegalovirus infection & $8.22 \times 10^{-3}$ & CDK6/EGFR/RUNX3/VEGFA \\
\hline hsa04014 & Ras signaling pathway & $8.80 \times 10^{-3}$ & EGF/EGFR/MET/VEGFA \\
\hline hsa04919 & Thyroid hormone signaling pathway & $1.08 \times 10^{-2}$ & NOTCH1/NOTCH2/NOTCH3 \\
\hline hsa05020 & Prion diseases & $1.29 \times 10^{-2}$ & RUNX3/NOTCH1 \\
\hline hsa04068 & FoxO signaling pathway & $1.38 \times 10^{-2}$ & EGF/EGFR/TNFSF10 \\
\hline hsa05162 & Measles & $1.38 \times 10^{-2}$ & CDK6/RUNX3/TNFSF10 \\
\hline hsa04934 & Cushing syndrome & $2.02 \times 10^{-2}$ & APC/CDK6/EGFR \\
\hline hsa05160 & Hepatitis C & $2.02 \times 10^{-2}$ & CDK6/EGF/EGFR \\
\hline hsa05144 & Malaria & $2.08 \mathrm{E}-02$ & RUNX3/MET \\
\hline hsa05164 & Influenza A & $2.48 \times 10^{-2}$ & RUNX3/PML/TNFSF10 \\
\hline hsa05202 & Transcriptional misregulation in cancer & $3.00 \times 10^{-2}$ & MET/PML/RUNX1 \\
\hline hsa05230 & Central carbon metabolism in cancer & $3.27 \times 10^{-2}$ & EGFR/MET \\
\hline hsa05221 & Acute myeloid leukemia & $3.27 \times 10^{-2}$ & PML/RUNX1 \\
\hline hsa05120 & $\begin{array}{l}\text { Epithelial cell signaling in Helicobacter } \\
\text { pylori infection }\end{array}$ & $3.27 \times 10^{-2}$ & EGFR/MET \\
\hline hsa05205 & Proteoglycans in cancer & $3.27 \times 10^{-2}$ & EGFR/MET/VEGFA \\
\hline hsa05211 & Renal cell carcinoma & $3.27 \times 10^{-2}$ & MET/VEGFA \\
\hline hsa04115 & p53 signaling pathway & $3.36 \times 10^{-2}$ & CDK6/MDM4 \\
\hline hsa04520 & Adherens junction & $3.36 \times 10^{-2}$ & EGFR/MET \\
\hline hsa04810 & Regulation of actin cytoskeleton & $3.51 \times 10^{-2}$ & APC/EGF/EGFR \\
\hline hsa05220 & Chronic myeloid leukemia & $3.55 \times 10^{-2}$ & CDK6/RUNX1 \\
\hline hsa04012 & ErbB signaling pathway & $4.28 \times 10^{-2}$ & EGF/EGFR \\
\hline hsa04540 & Gap junction & $4.47 \times 10^{-2}$ & EGF/EGFR \\
\hline hsa05323 & Rheumatoid arthritis & $4.55 \times 10^{-2}$ & RUNX3/VEGFA \\
\hline
\end{tabular}

APC, adenomatous polyposis coli protein. 
Table V. Significant GO terms enriched for genes in the competing endogenous RNA network.

\begin{tabular}{|c|c|c|c|c|}
\hline $\begin{array}{l}\mathrm{GO} \\
\text { term }\end{array}$ & ID & Description & $\begin{array}{l}\text { Adjusted } \\
\text { P-value }\end{array}$ & Gene ID \\
\hline $\mathrm{BP}$ & GO:0016055 & Wnt signaling pathway & $2.09 \times 10^{-5}$ & $\begin{array}{l}\text { APC/CTNND2/EGF/EGFR/MET/NOTCH1/ } \\
\text { RECK/RUNX1 }\end{array}$ \\
\hline $\mathrm{BP}$ & GO:0198738 & Cell-cell signaling by wnt & $2.09 \times 10^{-5}$ & $\begin{array}{l}\text { APC/CTNND2/EGF/EGFR/MET/NOTCH1/ } \\
\text { RECK/RUNX1 }\end{array}$ \\
\hline $\mathrm{BP}$ & GO:0030111 & Regulation of Wnt signaling pathway & $2.17 \times 10^{-5}$ & $\begin{array}{l}\text { APC/CTNND2/EGF/EGFR/NOTCH1/RECK/ } \\
\text { RUNX1 }\end{array}$ \\
\hline $\mathrm{BP}$ & GO:0060828 & $\begin{array}{l}\text { Regulation of canonical Wnt signaling } \\
\text { pathway }\end{array}$ & $7.32 \times 10^{-5}$ & APC/CTNND2/EGF/EGFR/NOTCH1/RECK \\
\hline $\mathrm{BP}$ & GO:0007050 & Cell cycle arrest & $7.32 \times 10^{-5}$ & APC/CDK6/MDM4/NOTCH1/NOTCH2/PML \\
\hline $\mathrm{BP}$ & GO:0045165 & Cell fate commitment & $9.34 \times 10^{-5}$ & APC/JAG1/NOTCH1/NOTCH2/NOTCH3/PML \\
\hline $\mathrm{BP}$ & GO:0060070 & Canonical Wnt signaling pathway & $1.31 \times 10^{-4}$ & APC/CTNND2/EGF/EGFR/NOTCH1/RECK \\
\hline $\mathrm{BP}$ & GO:0042176 & Regulation of protein catabolic process & $3.36 \times 10^{-4}$ & APC/EGF/EGFR/IL1B/MDM4/PML \\
\hline $\mathrm{BP}$ & GO:0048871 & Multicellular organismal homeostasis & $7.99 \times 10^{-4}$ & APC/EGFR/IL1B/MET/NOTCH1/VEGFA \\
\hline $\mathrm{BP}$ & GO:0033044 & Regulation of chromosome organization & $1.45 \times 10^{-3}$ & APC/BRD4/IL1B/PML/VEGFA \\
\hline $\mathrm{BP}$ & GO:0034329 & Cell junction assembly & $3.62 \times 10^{-3}$ & APC/CTNND2/RUNX1/VEGFA \\
\hline $\mathrm{BP}$ & GO:1901652 & Response to peptide & $3.98 \times 10^{-3}$ & APC/IL1B/JAG1/NOTCH1/TNFSF10 \\
\hline $\mathrm{BP}$ & GO:0071900 & $\begin{array}{l}\text { Regulation of protein serine/threonine } \\
\text { kinase activity }\end{array}$ & $4.29 \times 10^{-3}$ & APC/EGF/EGFR/IL1B/VEGFA \\
\hline $\mathrm{BP}$ & GO:0045930 & Negative regulation of mitotic cell cycle & $1.45 \times 10^{-3}$ & APC/EGFR/MDM4/PML \\
\hline $\mathrm{BP}$ & GO:0034330 & Cell junction organization & $4.78 \times 10^{-3}$ & APC/CTNND2/RUNX1/VEGFA \\
\hline $\mathrm{BP}$ & GO:0007043 & Cell-cell junction assembly & $5.19 \times 10^{-3}$ & APC/CTNND2/RUNX1 \\
\hline $\mathrm{BP}$ & GO:0010948 & Negative regulation of cell cycle process & $5.30 \times 10^{-3}$ & APC/MDM4/PML/SUZ12 \\
\hline $\mathrm{BP}$ & GO:0106106 & Cold-induced thermogenesis & $6.58 \times 10^{-3}$ & APC/NOTCH1/VEGFA \\
\hline BP & GO:0045216 & Cell-cell junction organization & $7.56 \times 10^{-3}$ & APC/CTNND2/RUNX1 \\
\hline MF & GO:0008013 & Beta-catenin binding & $3.47 \times 10^{-2}$ & APC/CTNND2 \\
\hline MF & GO:0031625 & Ubiquitin protein ligase binding & $3.47 \times 10^{-2}$ & APC/EGFR/PML \\
\hline MF & GO:0044389 & Ubiquitin-like protein ligase binding & $3.78 \times 10^{-2}$ & APC/EGFR/PML \\
\hline
\end{tabular}

Only terms enriched by adenomatous polyposis coli protein are listed. GO, Gene Ontology; BP, biological process; MF, molecular function; APC, adenomatous polyposis coli protein.

from the regulating synaptic membrane exocytosis (RIMS)2 gene. Thus, the function of hsa_circ_0005114 may be similar to that of RIMS2. Notably, Mukasa et al (32) observed that RIMS2 expression is significantly higher in normal brain tissues compared with glioblastoma. In addition, it was reported that RIMS2 mediates the cAMP-guanidine nucleotide exchange factor II pathway to promote incretin-potentiated insulin secretion $(33,34)$. The downregulation of RIMS2 may lead to the lower levels of insulin and the development of diabetes and obesity, as while $11.1 \%$ of patients with glioma (35) and $30 \%$ of those with brain tumor are diagnosed with obesity and insulin resistance/impaired glucose tolerance (36). Therefore, hsa_circ_0005114 may be involved in glioma by influencing the insulin secretion pathway, which was also predicted in the present function enrichment analysis. However, how hsa_circ_0005114 influences insulin secretion needs further investigation. A recent study proposed that high insulin index is positively associated with a high risk of glioma development (37). However, another study reported that decreased insulin receptor expression impairs cellular functions and represses orthotopic glioblastoma (38). These conflicting reports may be attributed to the dual roles of insulin (39).

In addition to insulin secretion mechanisms, the present study predicted that hsa_circ_0005114 may exert tumor suppressor functions by upregulating miR-142-3p and miR-590-5p, which subsequently inhibited the expression of APC. Studies have demonstrated that miR-590-5p is upregulated in liver (40), colorectal (41), gastric (42) and cervical cancer (43). miR-590-5p promotes cancer cell proliferation, invasion and therapy resistance by targeting FOXO1 (34), TGF- $\beta$ R2 (44), matrix metalloproteinases (41), reversion-inducing cysteine-rich protein with Kazal motifs (36) and cell adhesion molecule L1-like (43). Furthermore, a previous study also showed that miR-590-3p is upregulated in human glioma tissues (especially high grade) and radioresistant human glioblastoma cells (45). The use of anti-miR-590-3p suppresses cell viability, decreases colony formation capacity, increases the apoptotic rate and enhances the radiosensitivity. A luciferase reporter assay demonstrated that leucine-rich 
A

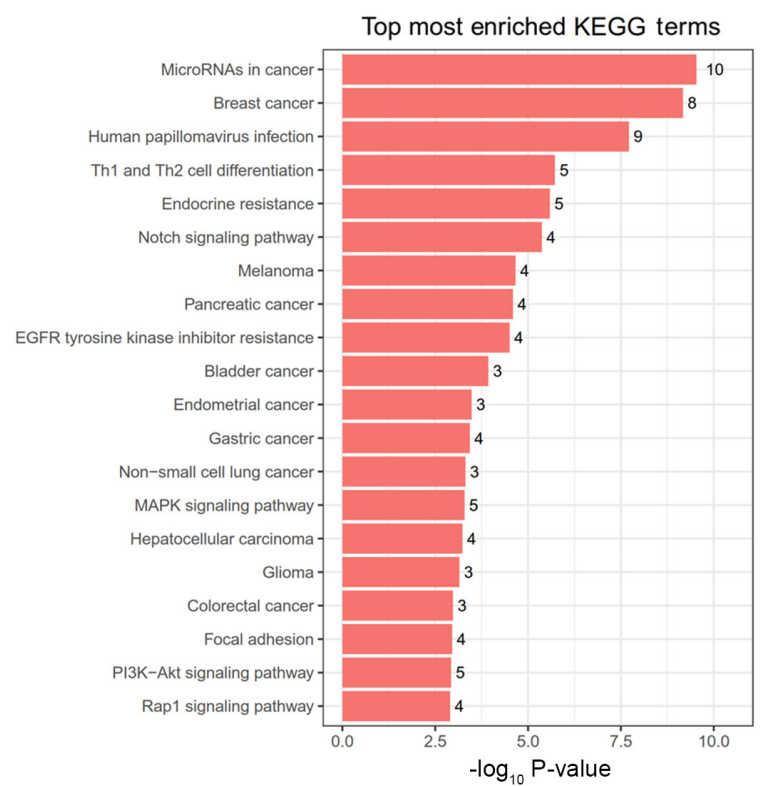

B

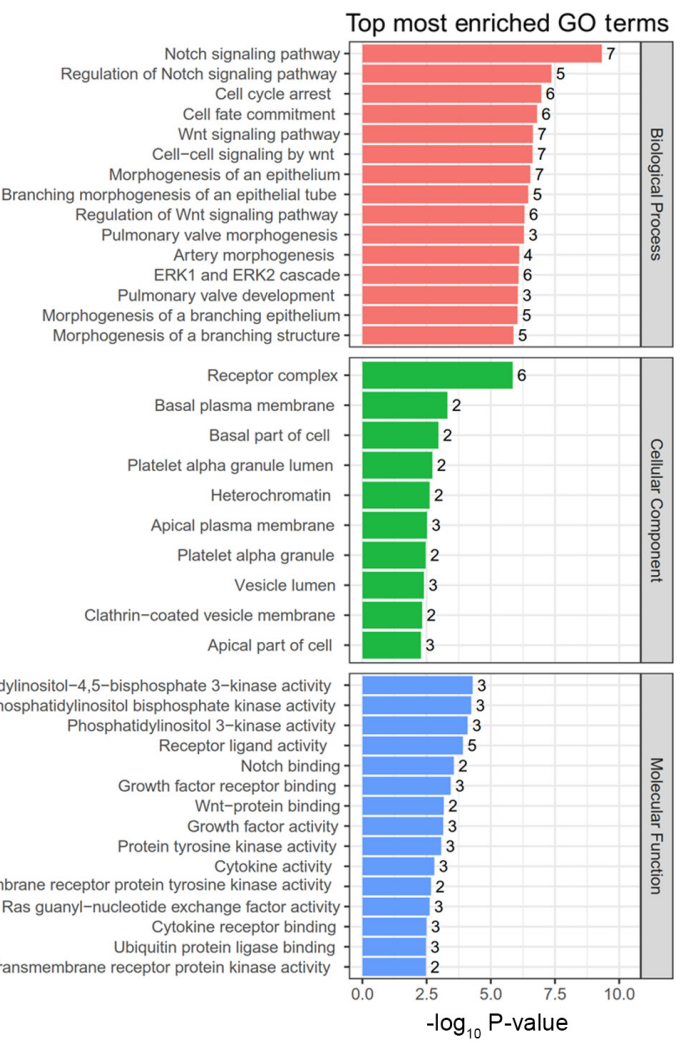

Figure 4. Function enrichment analysis for target genes of miRNAs. (A) Top 20 KEGG pathways and (B) top 15 biological process, cellular component and molecular function GO terms. KEGG, Kyoto Encyclopedia of Genes and Genomes; GO, Gene Ontology.

repeats and immunoglobulin-like domains protein 1 is the direct target of miR-590-3p. Similarly, other studies using quantitative PCR also detected that miR-142-3p expression is significantly upregulated in renal cell carcinoma (46) and nasopharyngeal carcinoma (47) compared with adjacent tissues. Downregulation of miR-142-3p significantly suppresses cell proliferation, migration and cell cycle progression, promotes apoptosis in vitro (46) and blocks tumor growth in a mouse model via upregulating its targeted genes, including suppressor of cytokine signaling 6 (47) and Ras-related C3 botulinum
Table VI. Overall survival-related mRNAs and miRNAs.

A, mRNA

\begin{tabular}{lcll}
\hline & $\begin{array}{c}\text { Cox regression } \\
\text { test }\end{array}$ & \multicolumn{1}{c}{ P-value } & FDR $(\mathrm{BH})$ \\
\hline SUZ12 & $7.823 \times 10^{-1}$ & $4.984 \times 10^{-05}$ & $2.991 \times 10^{-4}$ \\
VEGFA & $4.428 \times 10^{-1}$ & $1.000 \times 10^{-50}$ & $1.000 \times 10^{-49}$ \\
NOTCH3 & $5.14 \times 10^{-1}$ & $2.21 \times 10^{-11}$ & $6.64 \times 10^{-11}$ \\
RUNX3 & $5.539 \times 10^{-1}$ & $1.000 \times 10^{-38}$ & $1.000 \times 10^{-37}$ \\
CTNND2 & $-6.151 \times 10^{-1}$ & $1.000 \times 10^{-26}$ & $1.000 \times 10^{-25}$ \\
EGFR & $1.815 \times 10^{-1}$ & $2.405 \times 10^{-6}$ & $1.443 \times 10^{-5}$ \\
CDK6 & $4.738 \times 10^{-1}$ & $1.000 \times 10^{-17}$ & $1.000 \times 10^{-16}$ \\
PML & $8.536 \times 10^{-1}$ & $3.663 \times 10^{-12}$ & $2.198 \times 10^{-11}$ \\
BHLHE40 & $4.879 \times 10^{-1}$ & $3.331 \times 10^{-15}$ & $1.998 \times 10^{-14}$ \\
EGF & $3.093 \times 10^{-1}$ & $2.355 \times 10^{-9}$ & $7.065 \times 10^{-9}$ \\
NOTCH1 & $5.332 \times 10^{-1}$ & $1.000 \times 10^{-52}$ & $1.000 \times 10^{-52}$ \\
TNFSF10 & $3.983 \times 10^{-1}$ & $2.207 \times 10^{-13}$ & $6.621 \times 10^{-13}$ \\
RUNX1 & $5.332 \times 10^{-1}$ & $1.000 \times 10^{-52}$ & $1.000 \times 10^{-52}$ \\
MDM4 & $3.306 \times 10^{-1}$ & $1.131 \times 10^{-7}$ & $6.784 \times 10^{-7}$ \\
MET & $2.378 \times 10^{-1}$ & $1.000 \times 10^{-16}$ & $1.000 \times 10^{-16}$ \\
IL1B & $1.159 \times 10^{-1}$ & $1.164 \times 10^{-4}$ & $2.329 \times 10^{-4}$ \\
JAG1 & $6.596 \times 10^{-1}$ & $1.000 \times 10^{-33}$ & $1.000 \times 10^{-32}$ \\
APC & $-7.618 \times 10^{-1}$ & $1.000 \times 10^{-36}$ & $1.000 \times 10^{-35}$ \\
RECK & $4.158 \times 10^{-1}$ & $6.612 \times 10^{-3}$ & $1.984 \times 10^{-2}$
\end{tabular}

$\mathrm{B}$, miRNA

\begin{tabular}{lccc}
\hline RNA & $\begin{array}{c}\text { Cox regression } \\
\text { test }\end{array}$ & P-value & FDR (BH) \\
\hline hsa-miR-139-5p & $-1.909 \times 10^{-1}$ & $3.418 \times 10^{-3}$ & $1.025 \times 10^{-2}$ \\
hsa-miR-142-3p & $4.080 \times 10^{-1}$ & $6.370 \times 10^{-9}$ & $3.822 \times 10^{-8}$ \\
hsa-miR-217 & $2.006 \times 10^{-1}$ & $2.422 \times 10^{-6}$ & $1.453 \times 10^{-5}$ \\
hsa-miR-324-5p & $2.797 \times 10^{-1}$ & $6.083 \times 10^{-3}$ & $3.650 \times 10^{-2}$ \\
hsa-miR-346 & $-6.408 \times 10^{-1}$ & $2.317 \times 10^{-9}$ & $1.390 \times 10^{-8}$ \\
hsa-miR-590-5p & $5.611 \times 10^{-1}$ & $2.461 \times 10^{-7}$ & $7.384 \times 10^{-7}$ \\
hsa-miR-599 & $4.453 \times 10^{-1}$ & $9.471 \times 10^{-7}$ & $2.841 \times 10^{-6}$ \\
hsa-miR-615-3p & $9.771 \times 10^{-1}$ & $1.000 \times 10^{-8}$ & $1.000 \times 10^{-7}$ \\
hsa-miR-875-5p & $1.369 \times 10^{-1}$ & $5.275 \times 10^{-4}$ & $1.361 \times 10^{-3}$ \\
\hline
\end{tabular}

Only mRNAs and miRNAs significantly associated with overall survival $\mathrm{P} \leq 0.05$ are shown. FDR, False Discovery Rate; miRNA, micro RNA; APC, adenomatous polyposis coli protein; hsa, Homo sapiens; $\mathrm{BH}$, Benjamini and Hochberg.

toxin substrate 1 in colorectal cancer (48). High expression of miR-142-3p is correlated with histological differentiation and a poor prognosis for patients with esophageal squamous cell carcinoma (49). In line with the aforementioned findings, the present study reported that miR-590-5p and miR-142-3p were upregulated in glioblastoma tissues compared with normal controls and were associated with a worse prognosis. However, the targeted genes of these two miRNAs were not completely validated in cancer. It was predicted that APC, a regulator of the WNT signaling pathway (50), may be a direct target for 

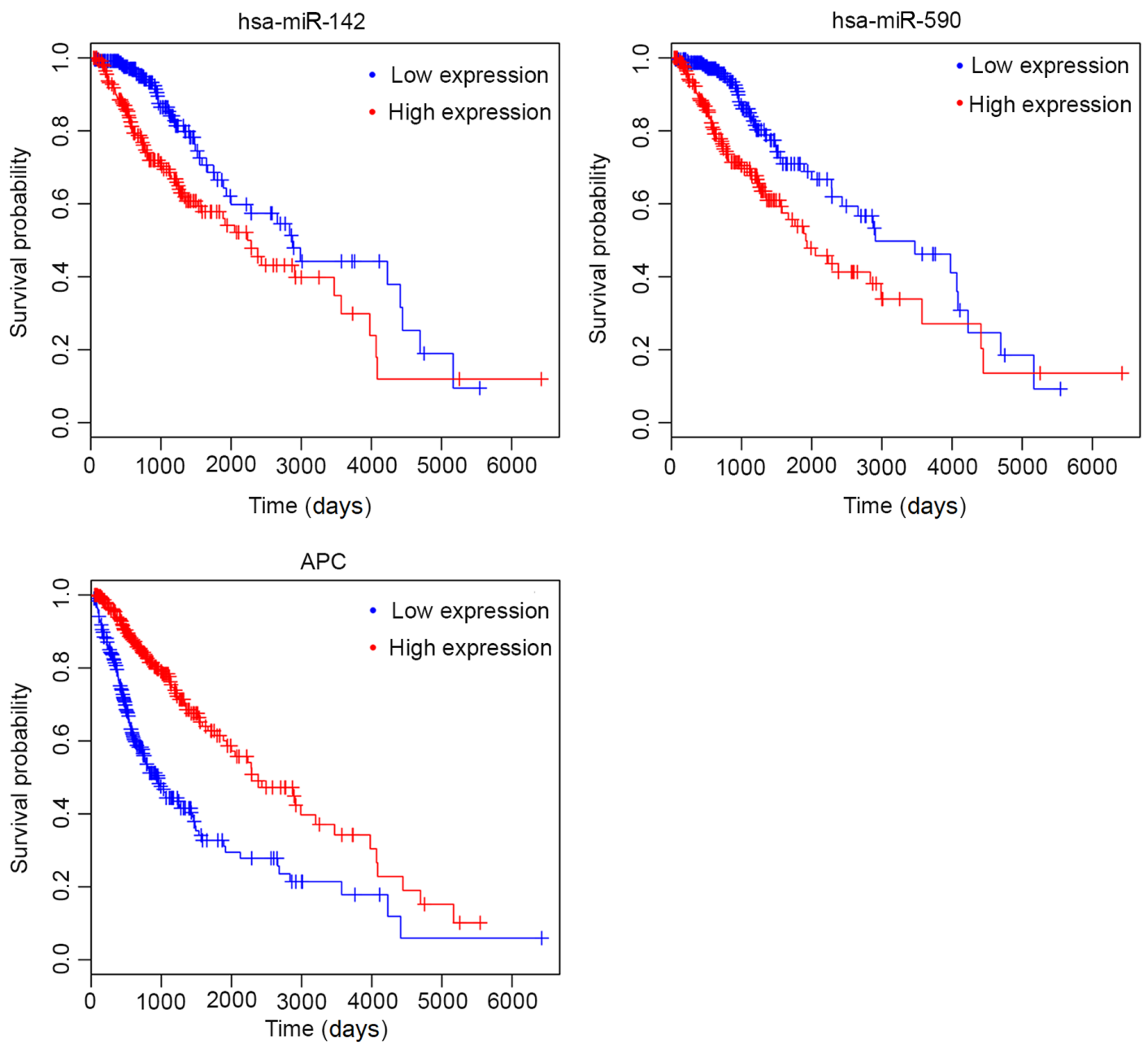

Figure 5. Association with overall survival of miRNAs and mRNA. Cox regression test was used as the statistical method to calculate the association. hsa, Homo sapiens.

both miR-590-5p and miR-142-3p. Their negative regulatory relationship was indirectly investigated by Naseri et al (51) who showed that delivery of anti-miR-142-3p by exosomes to breast cancer cells leads to a significant increase in APC mRNA levels. In addition, Wu et al (52) used dual-luciferase and western blot analysis in mesenchymal stem cells to demonstrate that miR-590-3p binds to the 3'UTR of APC mRNA. Notably, APC encodes a protein that acts as an antagonist of the Wnt signaling pathway (53), which is often activated in cancer (54). Thus, APC may be downregulated during carcinogenesis, which has been confirmed in various cancer types, including glioma (55-57). For example, Cole et al (55) reported that APC expression is decreased in pancreatic ductal adenocarcinoma tissues, and that APC siRNA treatment promotes cell proliferation and migration. Wang et al (56) reported that the expression of APC mRNA is significantly decreased in ovarian tumor cells and tissues compared with in normal ovarian cells and tissues. In addition, overexpression of APC induces increased apoptosis of ovarian tumor cells by decreasing the ATP binding cassette subfamily B member 1 (also known as multidrug resistance gene 1)/chemokine (C-X-C motif) ligand 1 signaling pathway. Zhang et al (57) demonstrated that APC expression is downregulated in colorectal cancer tissues, which is associated with the expression of miR-494.
Overexpression of miR-494 promotes colorectal cancer cell proliferation by inhibiting APC and consequently inducing Wnt/ $\beta$-catenin signaling. Western blot assays of Li et al (58) also suggested that miR-106a-5p reduces APC protein levels and upregulates target proteins of the Wnt/ $\beta$-catenin pathway, resulting in the invasion of glioblastoma cells (58). In accordance with these studies, the present study reported that APC expression was decreased in glioblastoma tissues compared with normal controls and high expression of APC was associated with a good prognosis. Functional enrichment analysis showed APC was involved in Wnt signaling pathway, cell fate commitment, and cell junction.

Overall, the present study suggested that hsa circ_0005114-miR-142-3p/miR-590-5p-APC ceRNA axes may be mechanisms for the development and progression of glioma. These axes may also have potential as novel targets for the treatment of glioma. However, additional in vitro and in vivo experiments, such as gene interaction experiments involving knockout/overexpression of hsa_circ_0005114-miR-142-3p/miR-590-5p-APC and their influence on tumor cells (using cell proliferation, apoptosis, invasion and migration assays) and tumor growth, are required to validate these conclusions in the future, and the lack of these experiments is a limitation of the present study. 


\section{Acknowledgements}

Not applicable.

\section{Funding}

The present study was funded by grants from The Natural Science Foundation of China (grant no. 81400404) and The Science and Technology of Jilin Province (grant no. 20200201470JC).

\section{Availability of data and materials}

The GSE86202, GSE92322, GSE103229 and GSE25632 datasets were downloaded from the Gene Expression Omnibus repository (http://www.ncbi.nlm.nih.gov/geo/). LinkedOmics and UALCAN database used the miRNA and mRNA sequencing data of The Cancer Genome Atlas (https://gdc-portal.nci.nih.gov/) for analysis.

\section{Authors' contributions}

BW, LW and JWZ conceived and designed the study. BW collected and analyzed the data. LW and JWZ contributed to the interpretation of data. BW and LW drafted the manuscript. JWZ revised the manuscript. All authors read and approved the final manuscript.

\section{Ethics approval and consent to participate}

Not applicable.

\section{Patient consent for publication}

Not applicable.

\section{Competing interests}

The authors declare that they have no competing interests.

\section{References}

1. Ryan CS, Juhn YJ, Kaur H, Wi CI, Ryu E, King KS and Lachance DH: Long-term incidence of glioma in Olmsted County, Minnesota, and disparities in postglioma survival rate: A population-based study. Neurooncol Pract 7: 288-298, 2020.

2. Sehmer EA, Hall GJ, Greenberg DC, O'Hara C, Wallingford SC, Wright KA and Green AC: Incidence of glioma in a northwestern region of England, 2006-2010. Neuro Oncol 16: 971-974, 2014.

3. Larjavaara S, Mäntylä R, Salminen T, Haapasalo H, Raitanen J, Jääskeläinen J and Auvinen A: Incidence of gliomas by anatomic location. Neuro Oncol 9: 319-325, 2007.

4. Rasmussen BK, Hansen S, Laursen RJ, Kosteljanetz M, SchultzH, Nørgård BM, Guldberg R and Gradel KO: Epidemiology of glioma: Clinical characteristics, symptoms, and predictors of glioma patients grade I-IV in the the Danish Neuro-Oncology Registry. J Neurooncol 135: 571-579, 2017.

5. Piñeros M, Sierra MS, Izarzugaza MI and Forman D: Descriptive epidemiology of brain and central nervous system cancers in Central and South America. Cancer Epidemiol 44 (Suppl 1): S141-S149, 2016.

6. Ebbesen KK, Kjems J and Hansen TB: Circular RNAs: Identification, biogenesis and function. Biochim Biophys Acta 1859: 163-168, 2016.

7. Yu CY and Kuo HC: The emerging roles and functions of circular RNAs and their generation. J Biomed Sci 26: 29, 2019.

8. Belousova EA, Filipenko ML and Kushlinskii NE: Circular RNA New regulatory molecules. Bull Exp Biol Med 164: 803-815, 2018.
9. Zhou J, Wang H, Chu J, Huang Q, Li G, Yan Y, Xu T, Chen J and Wang Y: Circular RNA hsa_circ_0008344 regulates glioblastoma cell proliferation, migration, invasion, and apoptosis. J Clin Lab Anal 32: e22454, 2018

10. Wang Y, Sui X, Zhao H, Cong L, Li Y, Xin T, Guo M and Hao W: Decreased circular RNA hsa_circ_0001649 predicts unfavorable prognosis in glioma and exerts oncogenic properties in vitro and in vivo. Gene 676: 117-122, 2018.

11. Zhan L, Mu Z, Yang M, Zhang T and Qian L: Elevation of circ-ITX1 upregulates interleukin 17 receptor D expression via sponging miR8a and facilitates cell progression in glioma. J Cell Biochem 120: 16495-16502, 2019.

12. Yi C, Li H, Li D, Qin X, Wang J, Liu Y, Liu Z and Zhang J: Upregulation of circular RNA circ_0034642 indicates unfavorable prognosis in glioma and facilitates cell proliferation and invasion via the miR-1205/BATF3 axis. J Cell Biochem 120: 13737-13744, 2019.

13. Xiong J, Wang T, Tang H, Lv Z and Liang P: Circular RNA circMAN2B2 facilitates glioma progression by regulating the miR-1205/S100A8 axis. J Cell Physiol 234: 22996-23004, 2019.

14. Duan X, Liu D, Wang Y and Chen Z: Circular RNA hsa circ_0074362 promotes glioma cell proliferation, migration, and invasion by attenuating the inhibition of miR-1236-3p on HOXB7 expression. DNA Cell Biol 37: 917-924, 2018.

15. Li X and Diao H: Circular RNA circ_0001946 acts as a competing endogenous RNA to inhibit glioblastoma progression by modulating miR-671-5p and CDR1. J Cell Physiol 234: 13807-13819, 2019.

16. Yuan Y, Li J, Xiang W, Liu Y and Mao Q: Analyzing the interactions of mRNAs, miRNAs, lncRNAs and circRNAs to predict competing endogenous RNA networks in glioblastoma. J Neurooncol 137: 493-502, 2018.

17. Zhu J, Ye J, Zhang L, Xia L, Hu H, Jiang H, Wan Z, Sheng F, Ma Y, Li W, et al: Differential expression of circular RNAs in glioblastoma multiforme and its correlation with prognosis. Transl Oncol 10: 271-279, 2017.

18. Glažar P, Papavasileiou P and Rajewsky N: circBase: A database for circular RNAs. RNA 20: 1666-1670, 2014.

19. Nikolayeva O and Robinson MD: edgeR for Differential RNA-seq and ChIP-seq analysis: An application to stem cell biology. Methods Mol Biol 1150: 45-79, 2014.

20. R Development Core Team. R: A language and environment for statistical computing. 2015. http://www.r-project.org/. Accessed, February 10, 2015.

21. Dudekula DB, Panda AC, Grammatikakis I, De S, Abdelmohsen K and Gorospe M: CircInteractome: A web tool for exploring circular RNAs and their interacting proteins and microRNAs. RNA Biol 13: 34-42, 2016.

22. Li JH, Liu S, Zhou H, Qu LH and Yang JH: Starbase v2.0: Decoding miRNA-ceRNA, miRNA-ncRNA and protein-RNA interaction networks from large-scale clip-seq data. Nucleic Acids Res 42 (Database issue): D92-D97, 2014.

23. Dweep H and Gretz N: miRWalk2.0: A comprehensive atlas of microRNA-target interactions. Nat Methods 12: 697, 2015.

24. Davis AP, Grondin CJ, Johnson RJ, Sciaky D, McMorran R, Wiegers J, Wiegers TC and Mattingly CJ: The Comparative Toxicogenomics Database: Update 2019. Nucleic Acids Res 47: D948-D954, 2019.

25. Kohl M, Wiese S and Warscheid B: Cytoscape: Software for visualization and analysis of biological networks. Methods Mol Biol 696: 291-303, 2011

26. Yu G, Wang LG, Han Y and He QY: clusterProfiler: An R package for comparing biological themes among gene clusters. OMICS 16: 284-287, 2012.

27. Ferreira JA: The Benjamini-Hochberg method in the case of discrete test statistics. Int J Biostat 3: 11, 2007.

28. Vasaikar SV, Straub P, Wang J and Zhang B: LinkedOmics: Analyzing multi-omics data within and across 32 cancer types. Nucleic Acids Res 46 (Database issue): D956-D963, 2017.

29. Chandrashekar DS, Bashel B, Balasubramanya SAH, Creighton CJ, Ponce-Rodriguez I, Chakravarthi BVSK and Varambally S: UALCAN: A portal for facilitating tumor subgroup gene expression and survival analyses. Neoplasia 19: 649-658, 2017.

30. Zhang W, Zhang J, Hoadley K, Kushwaha D, Ramakrishnan V, Li S, Kang C, You Y, Jiang C, Song SW, et al: miR-181d: A predictive glioblastoma biomarker that downregulates MGMT expression. Neuro Oncol 14: 712-719, 2012. 
31. Lu J,Zhang PY, Li P, Xie JW, Wang JB, Lin JX, Chen QY, Cao LL, Huang CM and Zheng CH: Circular RNA hsa_circ_0001368 suppresses the progression of gastric cancer by regulating miR-6506-5p/FOXO3 axis. Biochem Biophys Res Commun 512: 29-33, 2019.

32. Mukasa A, Ueki K, Ge X, Ishikawa S, Ide T, Fujimaki T, Nishikawa R, Asai A, Kirino T and Aburatani H: Selective expression of a subset of neuronal genes in oligodendroglioma with chromosome 1p loss. Brain Pathol 14: 34-42, 2010.

33. Kashima Y, Miki T, Shibasaki T, Ozaki N, Miyazaki M, Yano H and Seino S: Critical role of cAMP-GEFII-Rim2 complex in incretin-potentiated insulin secretion. J Biol Chem 276 46046-46053, 2001

34. Fujimoto K, Shibasaki T, Yokoi N, Kashima Y, Matsumoto M, Sasaki T, Tajima N, Iwanaga T and Seino S: Piccolo, a Ca ${ }^{2+}$ sensor in pancreatic beta-cells. Involvement of cAMP-GEFII.Rim2. Piccolo complex in cAMP-dependent exocytosis. J Biol Chem 277: 50497-50502, 2002.

35. Sani I and Albanese A: Endocrine long-term follow-up of children with neurofibromatosis type 1 and optic pathway glioma. Horm Res Paediatr 87: 178-188, 2017.

36. Masanori A, Takayoshi T, Koji M, Yumi A, Ken-Ichi S and Hironobu S: Prevalence of obesity, hyperlipemia and insulin resistance in children with suprasellar brain tumors. Clin Pediatr Endocrinol 16: 1-9, 2007.

37. Anjom-Shoae J, Shayanfar M, Mohammad-Shirazi M, Sadeghi O, Sharifi G, Siassi F and Esmaillzadeh A: Dietary insulin index and insulin load in relation to glioma: Findings from a case-control study. Nutr Neurosci 29: 1-9, 2019.

38. Gong Y, Ma Y, Sinyuk M, Loganathan S, Thompson RC, Sarkaria JN, Chen W, Lathia JD, Mobley BC, Clark SW and Wang J: Insulin-mediated signaling promotes proliferation and survival of glioblastoma through Akt activation. Neuro Oncol 18 : 48-57, 2016.

39. Yang BC, Wang YS, Wang $\mathrm{CH}$, Lin $\mathrm{HH}$, Tang MJ and Yang TL: Transient apoptosis elicited by insulin in serum-starved glioma cells involves Fas/Fas-L and Bcl-2. Cell Biol Int 23: 533-540, 1999.

40. Jia G, Tang Y, Deng G, Fang D, Xie J, Yan L and Chen Z: miR-590-5p promotes liver cancer growth and chemotherapy resistance through directly targeting FOXO1. Am J Trans Res 11: 2181-2193, 2019.

41. Kim CW, Oh ET, Kim JM, Park JS, Lee DH, Lee JS, Kim KK and Park HJ: Hypoxia-induced microRNA-590-5p promotes colorectal cancer progression by modulating matrix metalloproteinase activity. Cancer Lett 416: 31-41, 2018.

42. Shen B, Yu S, Zhang Y, Yuan Y, Li X, Zhong J and Feng J: miR-590-5p regulates gastric cancer cell growth and chemosensitivity through RECK and the AKT/ERK pathway. Onco Targets Ther 9: 6009-6019, 2016.

43. Chu Y, Ouyang Y, Wang F, Zheng A, Bai L, Han L, Chen Y and Wang H: MicroRNA-590 promotes cervical cancer cell growth and invasion by targeting CHL1. J Cell Biochem 115: 847-853, 2014.

44. Jiang X, Xiang G, Wang Y, Zhang L, Yang X, Cao L, Peng H, Xue $\mathrm{P}$ and Chen D: MicroRNA-590-5p regulates proliferation and invasion in human hepatocellular carcinoma cells by targeting TGF- $\beta$ RII. Mol Cells 33: 545-551, 2012.
45. Chen L, Wang W, Zhu S, Jin X, Wang J, Zhu J and Zhou Y: MicroRNA-590-3p enhances the radioresistance in glioblastoma cells by targeting LRIG1. Exp Ther Med 14: 1818-1824, 2017.

46. Li Y, Chen D, Jin L, Liu J, Li Y, Su Z, Qi Z, Shi M, Jiang Z, Yang S, et al: Oncogenic microRNA-142-3p is associated with cellular migration, proliferation and apoptosis in renal cell carcinoma. Oncol Lett 11: 1235-12341, 2016.

47. Qi X, Li J, Zhou C, Lv C and Tian M: miR-142-3p suppresses SOCS6 expression and promotes cell proliferation in nasopharyngeal carcinoma. Cell Physiol Biochem 36: 1743-1752, 2015.

48. Gao X, Xu W, Lu T, Zhou J, Ge X and Hua D: MicroRNA-142-3p promotes cellular invasion of colorectal cancer cells by activation of RAC1. Technol Cancer Res Treat 17: 1533033818790508, 2018.

49. Lin RJ, Xiao DW, Liao LD, Chen T, Xie ZF, Huang WZ, Wang WS, Jiang TF, Wu BL, Li EM and Xu LY: miR-142-3p as a potential prognostic biomarker for esophageal squamous cell carcinoma. J Surg Oncol 105: 175-182, 2012.

50. Hankey W, Frankel WL and Groden J: Functions of the APC tumor suppressor protein dependent and independent of canonical WNT signaling: Implications for therapeutic targeting. Cancer Metastasis Rev 37: 159-172, 2018.

51. NaseriZ, Oskuee RK,Jaafari MR and ForouzandehMoghadam M: Exosome-mediated delivery of functionally active miRNA-142-3p inhibitor reduces tumorigenicity of breast cancer in vitro and in vivo. Int J Nanomedicine 13: 7727-7747, 2018.

52. Wu S, Liu W and Zhou L: miR-590-3p regulates osteogenic differentiation of human mesenchymal stem cells by regulating APC gene. Biochem Biophys Res Commun 478: 1582-1587, 2016.

53. Saito-Diaz K, Benchabane H, Tiwari A, Tian A, Li B, Thompson JJ, Hyde AS, Sawyer LM, Jodoin JN, Santos E, et al: APC inhibits ligand-independent Wnt signaling by the clathrin endocytic pathway. Dev Cell 44: 566-581.e8, 2018.

54. Ghosh N, Hossain U, Mandal A and Sil PC: The Wnt signaling pathway: A potential therapeutic target against cancer. Ann N Y Acad Sci 1443: 54-74, 2019.

55. Cole JM, Simmons K and Prosperi JR: Effect of adenomatous polyposis coli loss on tumorigenic potential in pancreatic ductal adenocarcinoma. Cells 8: 1084, 2019.

56. Wang Y, Cao C, Fang D and Hu Y: Role of APC-mediated MDR-1/CLCX-1 signaling pathway in ovarian tumors. J Biol Regul Homeost Agents 32: 529-536, 2018.

57. Zhang Y, Guo L, Li Y, Feng GH, Teng F, Li W and Zhou Q: MicroRNA-494 promotes cancer progression and targets adenomatous polyposis coli in colorectal cancer. Mol Cancer 17: 1, 2018.

58. Li D, Wang Z, Chen Z, Lin L, Wang Y, Sailike D, Luo K, Du G, Xiang X and Jiafu GD: MicroRNA-106a-5p facilitates human glioblastoma cell proliferation and invasion by targeting adenomatosis polyposis coli protein. Biochem Biophys Res Commun 481: 245-250, 2016 International (CC BY-NC-ND 4.0) License. 